\title{
The risk of hyperbilirubinemia in term neonates after placental transfusion - a randomized-blinded controlled trial
}

\author{
Joanna P. Seliga-Siwecka®, Joanna Puskarz-Gasowska, Justyna Tolloczko \\ Department of Neonatology and Intensive Care Unit, Faculty of Medicine, Medical University of Warsaw, Poland
}

\begin{abstract}
:
Objective: We aimed to demonstrate non-inferiority of delayed cord clamping (DCC) and cord milking (CM) in comparison to early cord clamping $(\mathrm{ECC})$ in the incidence of hyperbilirubinemia requiring phototherapy.

Material and methods: 467 of maternal-foetal dyads were screened for eligibility. 389 term infants, of breastfeeding, non-smoking mothers were randomized to receive ECC $(<40 \mathrm{~s})$, DCC (1-2 min) or CM (4 times towards the neonate). The primary outcome was defined as hyperbilirubinemia requiring phototherapy.

Results: 307 patients were included in the analysis. CM did not increase the risk of phototherapy RR $11.2795 \% \mathrm{Cl}(0.80$; 2.04). Similar results were achieved when comparing DCC and ECC, RR $1.2995 \% \mathrm{Cl}(0.82 ; 2.05)$. This was also true for CM vs DCC, RR $0.9995 \% \mathrm{Cl}(0.64 ; 1.52)$. The prevalence of total serum bilirubin (TSB) at $24-48$ hours was $10.8 \mathrm{mg} / \mathrm{dL} ; 10.33 \mathrm{mg} / \mathrm{dL}$ and 11.39 in ECC, CM and DCC group respectively. Transcutaneous bilirubin (TCB) levels at $24-48 \mathrm{~h}$ were $7.58 \mathrm{mg} / \mathrm{dL}$, $7.89 \mathrm{mg} / \mathrm{dL}$ and $7.60 \mathrm{mg} / \mathrm{dL}$ in the ECC, CM and DCC respectively. None of the neonates met exchange transfusion criteria or symptomatic polycythaemia.

Conclusions: Our study suggests that placental transfusion is not associated with hyperbilirubinemia requiring phototherapy or exchange transfusion.
\end{abstract}

Key words: jaundice; hyperbilirubinemia; neonate; placental transfusion

\section{INTRODUCTION}

Delayed cord clamping (DCC), also known as expectant or physiological cord clamping, has been a subject of extensive research for the last couple of years. It involves clamping the cord when pulsation has ceased or at least after 30-60 seconds, allowing for foetal blood transfer from the placenta to the infant [1]. Cord milking (CM), an alternative method, involves milking the cord towards the infants 4 times. These interventions, labeled as placental transfusion, can provide the infant with respectively up to $30 \%$ and $60 \%$ additional blood volume and red blood cells [2]. Numerous neonatal benefits of DCC have been suggested including increased haemoglobin and ferritin levels both at birth and at longer term [3]. Nevertheless, systematic reviews of DCC versus early or immediate cord clamping (ECC) reveal that it may also contribute to other neonatal outcomes including polycythaemia and hyperbilirubinemia [2-4].

A Cochrane review published in 2008 determined maternal and neonatal effects of different policies of cord clamp- ing timing during the third stage of labour in term infants [3]. Eight trials examined jaundice requiring phototherapy. However, evidence for decreased risk of jaundice requiring phototherapy in the ECC group was based upon one unpublished trial. The variable did not reach statistical significance if this one trial was removed from the analysis. No difference was detected for polycythaemia. A meta-analysis published by Hutton provides contradictory results [5]. No significant differences were found between groups. Recently, a large study from Australia was published where the authors found that in preterm infants DCC did not increase the risk of hyperbilirubinemia [6]. Nevertheless, a systematic review from the same group showed that there was a significant increase in polycythaemia and jaundice [7]. It is important to note, that none of the reviews distinguished between DCC and $C M$, as opposed interventions to ECC. Based on numerous proven benefits, placental transfusion has been widely adopted in perinatal centres (personal communication) and is now part of both Polish and international guidelines on 
neonatal resuscitation [1]. A retrospective study published by Yang et al. has shown that implementing placental transfusion as a unit protocol did not increase the number of infants requiring phototherapy [8]. We are the first to report a prospective double blinded randomised controlled trial.

\section{Objectives}

The primary end point of this study was to evaluate if placental transfusion (delayed cord clamping or cord milking) increases the risk hyperbilirubinemia requiring phototherapy in term infants.

\section{MATERIAL AND METHODS}

We conducted a trial using observer blinded, balanced randomization [1:1:1], and included 3 parallel groups. We planned to demonstrate the non-inferiority of placental transfusion in comparison to ECC in regard to the incidence of hyperbilirubinemia requiring phototherapy.

Eligible participants were maternal-foetal dyads, in labour at 37-42 weeks of gestation. We recruited non-smoking mothers, willing to return for follow up visits, who declared to breastfeed for at least 6 months. Exclusion criteria included iso-immune haemolytic disease, sepsis, maternal Gilbert syndrome, birth asphyxia, need for resuscitation and serious maternal haemorrhage during delivery.

The study took place at a level III teaching hospital with approximately 3500 deliveries per year $(2000>37$ weeks of gestation) and 67 neonatal beds. During the trial, the local protocol on cord clamping had not been yet adjusted to the new International Liaison Committee on Resuscitation (ILCOR) guidelines [1].

A member of the recruitment team approached the mothers prior to delivery in the labour ward. He/she explained the study and obtained written consent for participating in the trial. The patient's medical record number (MRN) was registered on a secure web-based platform and demographic data was recorded. Maternal-foetal dyads, prior to delivery, were randomly assigned to receive ECC, DCC or CM. The midwife or obstetrician was informed about the allocated intervention preceding the delivery of the shoulders (spontaneous vaginal delivery), or head (caesarean section).

During vaginal deliveries midwifes were asked to maintain the infant at least $10 \mathrm{~cm}$ above the uterus until the cord is clamped, as this has been found to be most effective in obtaining adequate blood volumes $[9,10]$. In cases of caesarean sections, the baby was placed on the mother's laps and swaddled in sterile towels to prevent heat loss. In the ECC and DCC group, the recruiter informed the team when 30 seconds or 2 minutes had passed. The attending labour ward staff clamped the cord afterwards.

If CM was applied the baby was placed about $20 \mathrm{~cm}$ below the level of the placenta, between the mother's thighs (during a vaginal delivery) or at the side of the mother, swaddled in sterile towels (during a caesarean delivery). Before starting the trial we piloted this procedure previously described by Rabe et al. [11]. We assumed that the placental would contain about $40 \%$ of total circulating foetal blood with about $10-15 \mathrm{~mL}$ present for placental transfusion in the umbilical vein, both during a vaginal and caesarean delivery [12]. If the cord was milked once at the speed of $20 \mathrm{~cm} / 2 \mathrm{sec}$ onds, we were able to transfer approximately $10-13 \mathrm{~mL}$ of blood to the neonate. Under the assumption that the cord vein will rapidly refill itself, we assumed that milking the cord four times would give us $30-40 \mathrm{~mL}$ of blood. This amount is similar to the quantity of blood transfused to the neonate during DCC [11]. A member of the delivery team (vaginal delivery) or operating team (caesarean section) held the cord at the level of the introitus or caesarean wound and milked the cord four times towards the neonate counting out loud. The cord was clamped after the fourth milking.

The primary endpoint with respect to the risk of hyperbilirubinemia, was the number of neonates requiring phototherapy or exchange transfusion as defined by the American Academy of Paediatrics (AAP) guidelines [13]. Infants were assessed for the risk of developing hyperbilirubinemia or the need for exchange transfusion based on gestational age and risk factors as defined by Bhutani et al. [13]. If levels exceeded predefined thresholds, phototherapy was applied. In infants receiving treatment, bilirubin levels were reordered every 48 hours as per unit protocol.

The secondary objectives were to determine if DCC or CM compared to ECC, influenced the risk for polycythaemia during the first week of life, congenital anaemia, and readmission for hyperbilirubinemia during the first two weeks of life. Polycythaemia was defined as venous haematocrit of more than $65 \%$ or venous haemoglobin above $22 \mathrm{~g} / \mathrm{dL}$. Congenital anaemia was diagnosed in babies with cord haemoglobin $<12.5 \mathrm{~g} / \mathrm{dL}$ [14]. The decision for readmission for hyperbilirubinemia was based on the AAP guidelines for initiating phototherapy [15].

Sample sizes were estimated based on proportions of neonates in need of phototherapy between groups reported by McDonald ( $8.8 \%$ and $4.1 \%$ of neonates with jaundice in the DCC and ECC group respectively) [16]. Initially we calculated that, to detect a higher prevalence of neonates requiring phototherapy in the DCC group with one-tailed a value of 0.05 and $80 \%$ power, 380 children should be enrolled in every group (1140 children in total). Unfortunately, we were only able to secure limited funds to complete the study, which forced us to significantly decrease the number of participants.

The patient's MRN was registered by one of the recruiters on a secure web-based platform, initial demographic data was recorded, and a random computer-generated treatment was allocated. Randomization was created by Blockrand 
software (R Foundation for Statistical Computing, Vienna, Austria). Block randomization by delivery mode was applied. Patients were randomly assigned to ECC, DCC or CM in a 1:1:1 ratio. The block size was variable and concealed until primary endpoint analyses.

Due to the nature of the intervention, we could not take any measures to blind the intervention, however caregivers deciding on the initiation of phototherapy were blinded to intervention allocation. To prevent bias, members of the recruitment team did not participate in the further care of neonates on postnatal wards.

Venous cord blood samples were collected for each maternal-neonate dyad. Non-invasive transcutaneous bilirubin levels (TcB) were evaluated using a bilirubinometer (Bilicheck, Philips, Andover, MA, USA) every 24 hours during evening nursing rounds until discharge. The bilirubinometer was calibrated prior to every measurement, as per manufacture's recommendation. Venous samples for total serum bilirubin (TsB) were ordered on the discretion of the attending physician or if TcB extended recommended levels [17]. Olympus AU 480 (Beckman Coulter, Fullerton, CA, USA) was used to measure TsB. The analyser's calibration was checked with appropriate controls as per product guidelines.

Statistical comparison of baseline demographics between groups was performed using chi-squared test for frequency data. For continuous variables t-test was used when Shapiro-Wilk test did not rejected assumption of normality, otherwise Wilcoxon rank sum test was used. For the assessment of primary and secondary endpoints, 95\% Cl and $p$ values for frequency data were calculated assuming normal approximation of a binomial distribution. To assess phototherapy duration and hospitalization duration, a comparison Wilcoxon rank sum test was used.

The local Bioethics Committee approved the study. Our study is registered with ClinicalTrials.gov.

\section{RESULTS}

Recruitment was conducted from January 2014 to June 2016. We approached 467 eligible women in labour and invited them to take part in the trial. Seventy-eight women either declined participation or were not enrolled for operative reasons. The remaining 389 maternal-foetal dyads were randomly assigned to 3 interventions (Fig. 1).

Compliance with allocated treatment was $96.9 \%, 91.5 \%$ and $90.1 \%$ in the ECC, CM and DCC groups respectively. Reasons for allocation deviation are presented in Fig1. Study groups were similar with respect to demographic and clinical variables (Tab. 1).

\section{Primary outcomes}

The primary analysis was planned as modified intention-to-treat (mITT) and included all patients, who were randomly assigned to procedures and passed inclusion and exclusion criteria. The initially planned number of patients was not achieved due to funding limitations. Forty-one infants were excluded from the analysis, including 26 neonates lost for follow-up or with incomplete data before assessment of eligibility criteria or reaching the primary endpoint (Fig. 1). In these cases, the reason for attrition was not known, which can lead to the bias.

The number of children who were randomized and passed inclusion/exclusion criteria was 109, 99 and 109 in ECC, CM and DCC group, respectively (Fig. 1).

To perform mITT analysis of the primary outcome, a single imputation method was planned to substitute missing data. Given the size of groups, the imputation could have substantial impact on the proportion of phototherapy which can lead to bias on estimated parameters but has little effect on the low power of the non-inferiority test, therefore per protocol primary outcome analysis was performed.

The percentage of neonates requiring phototherapy did not differ significantly between the ECC, CM and DCC group ( $23 \%, 29 \%$ and $29 \%$, respectively). CM compared to ECC did not increase the risk of phototherapy, with a mean difference between two treatment arms of $6.2 \%$ and RR $1.2795 \% \mathrm{Cl}$ $(0.80 ; 2.04)$. Similar results were achieved when comparing DCC and ECC, with a mean difference of $6.6 \%$ and RR $1.2995 \%$ $\mathrm{Cl}(0.82 ; 2.05)$. This was also true for both methods of placental transfusion (CM vs DCC) RR $0.9995 \% \mathrm{Cl}(0.64 ; 1.52)$ (Tab. 2).

For the non-inferiority analysis, the non-inferiority margin was set at $4 \%$ as the largest difference being clinically acceptable. We did not find published data regarding neonatal jaundice requiring phototherapy in neonates receiving cord milking, thus we assumed the same non-inferiority margin for all comparisons.

The non-inferiority of both therapies was assessed based on whether the pre-specified treatment effect falls within $95 \%$ one-tailed interval for the treatment effect, which is the same as the upper limit of a two-tailed $90 \% \mathrm{Cl}$. The non-inferiority margin was within $90 \%$ of the two-tailed $\mathrm{Cl}$ for the absolute risk difference between CM and ECC (-3.9; 16.3\%) and for the absolute risk difference between DCC and ECC $(-3.3 \%$; $16.5 \%)$. Given that obtaining adequate sample size in our study was infeasible and the small statistical power of non-inferiority test ( $12 \%$ for CM vs ECC, and $13 \%$ for DCC vs ECC), a conclusive decision cannot be made about non-inferiority of $C M$ and DCC procedures with respect to ECC.

\section{Secondary outcomes}

The prevalence of total serum bilirubin (TSB) at 2448 hours was in all study groups. The average time (hours) of phototherapy was 58.0 in the ECC group, 49.1 in the CM group and 63.4 in the DCC group (Tab. 2). None of the patients had asymptomatic or symptomatic polycythaemia 


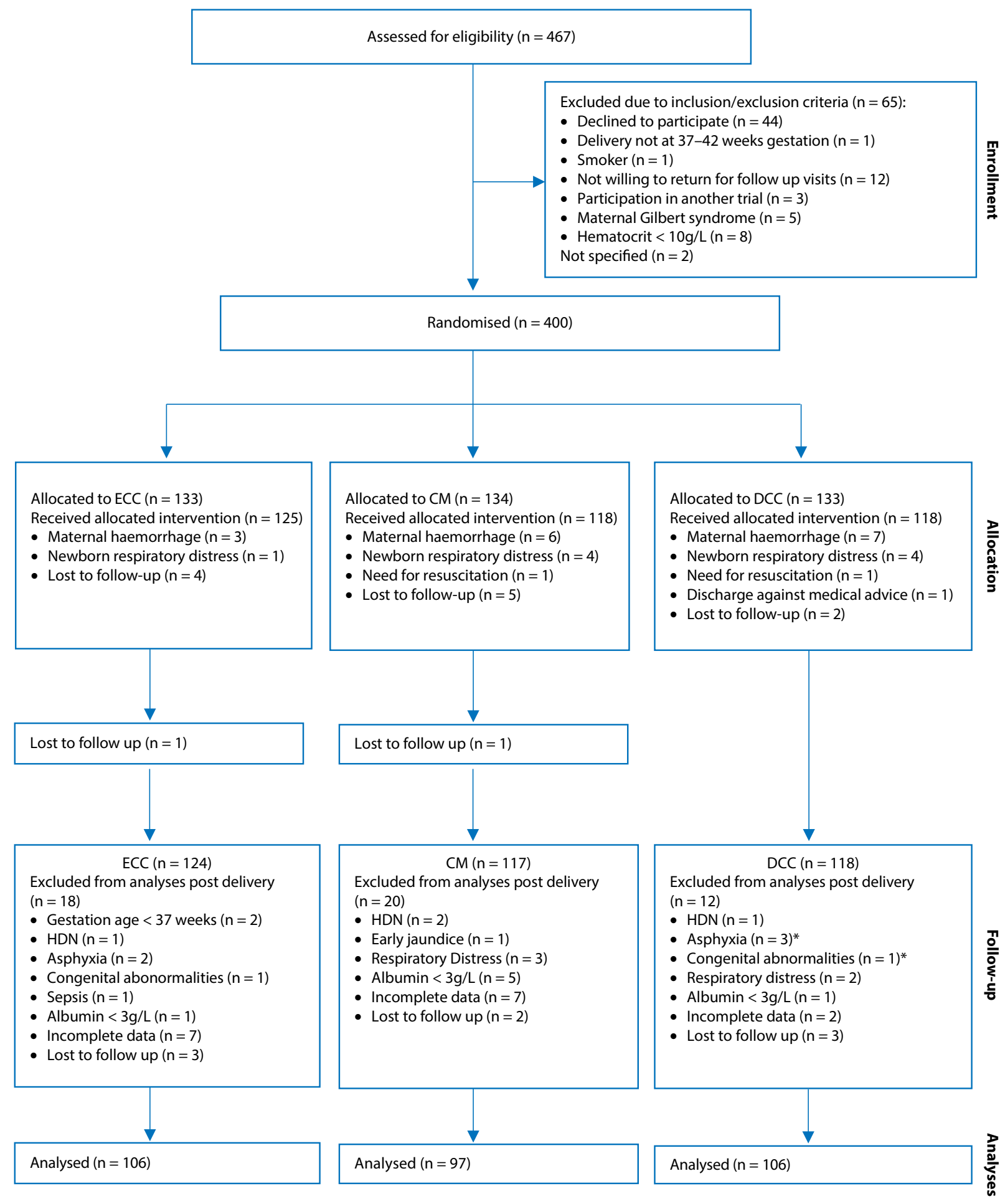

Figure 1. Randomization flow chart

(defined as a haematocrit $>65 \%$ ). One infant in the ECC group ( $n=26)$, none in the CM group $(n=24)$ and 5 neonates in the DCC group $(n=39)$ presented with venous haemoglobin above $22 \mathrm{~g} / \mathrm{dL}$ (Tab. 2). One patient per group (CM and $\mathrm{DCC}$ ) required a second course of phototherapy prior to discharge. On average, infants from ECC group were dis- charged home at 4.1 days, while infants from CM and DCC groups were hospitalized for 4.4 and 4.5 days, respectively.

\section{DISCUSSION}

A meta-analyses estimated a significant $47 \%$ reduction in the risk of anaemia and $33 \%$ reduction in the risk of iron 


\begin{tabular}{|c|c|c|c|c|c|c|}
\hline & $\begin{array}{l}\text { ECC } \\
(n=106)\end{array}$ & $\begin{array}{l}\text { CM } \\
(n=97)\end{array}$ & $\begin{array}{l}\text { DCC } \\
(n=106)\end{array}$ & $\begin{array}{l}\text { CM vs ECC } \\
\text { P Value }\end{array}$ & $\begin{array}{l}\text { DCC vs ECC } \\
\text { P Value }\end{array}$ & $\begin{array}{l}\text { CM vs DCC } \\
\text { P Value }\end{array}$ \\
\hline \multicolumn{7}{|l|}{ Gender, \% } \\
\hline Male & $49.1 \%$ & $43.3 \%$ & $48.1 \%$ & 0.411 & 0.891 & 0.492 \\
\hline Female & $50.9 \%$ & $56.7 \%$ & $51.9 \%$ & & & \\
\hline Mean (SD) birth weight, $g$ & $3503(459)$ & $3485(399)$ & $3551(546)$ & 0.767 & 0.493 & 0.328 \\
\hline Mean (SD) gestational age, wk & $39.11(0.99)$ & $39.00(1.01)$ & $39.01(1.07)$ & 0.538 & 0.448 & 0.884 \\
\hline \multicolumn{7}{|l|}{ Method of childbirth, \% } \\
\hline Spontaneous vaginal delivery & $53.8 \%$ & $43.3 \%$ & $48.1 \%$ & 0.136 & 0.410 & 0.492 \\
\hline Caesarean section & $46.2 \%$ & $56.7 \%$ & $51.9 \%$ & & & \\
\hline Median APGAR1 (Q1, Q3) & $10(10,10)$ & $10(10,10)$ & $10(10,10)$ & & & \\
\hline Proportion APGAR1 < 10\% & $8.5 \%$ & $8.2 \%$ & $5.7 \%$ & 0.950 & 0.422 & 0.468 \\
\hline Median APGAR5 (Q1, Q3) & $10(10,10)$ & $10(10,10)$ & $10(10,10)$ & & & \\
\hline Proportion APGAR5 $<10 \%$ & $6.6 \%$ & $5.2 \%$ & $6.6 \%$ & 0.662 & 1.000 & 0.662 \\
\hline Mean (SD) cord blood Hb, g/dL & $16.80(1.93)$ & $16.33(2.22)$ & $16.62(2.22)$ & 0.072 & 0.263 & 0.406 \\
\hline Mean (SD) cord blood bilirubin, mg/dL & $2.07(0.63)$ & $2.06(0.55)$ & $1.93(0.49)$ & 0.809 & 0.097 & 0.162 \\
\hline Maximum value & 4.4 & 3.54 & 3.82 & & & \\
\hline Mean (SD) transcutaneous bilirubin (first 24 hours), mg/dL & $4.20(2.06)$ & $4.19(1.71)$ & 3.99 (1.87) & 0.995 & 0.581 & 0.499 \\
\hline Range (min; max) & {$[0 ; 10]$} & {$[1.2 ; 9.1]$} & {$[0 ; 8.7]$} & & & \\
\hline Mean (SD) cord albumin, g/dL & $3.50(0.25)$ & $3.48(0.24)$ & $3.44(0.23)$ & 0.644 & 0.083 & 0.197 \\
\hline
\end{tabular}

ECC — early cord clamping; CM — cord milking; DCC — delayed cord clamping; IQR — interquadral; SD — standard deviation; $\mathrm{Hb}$ - hemoglobin

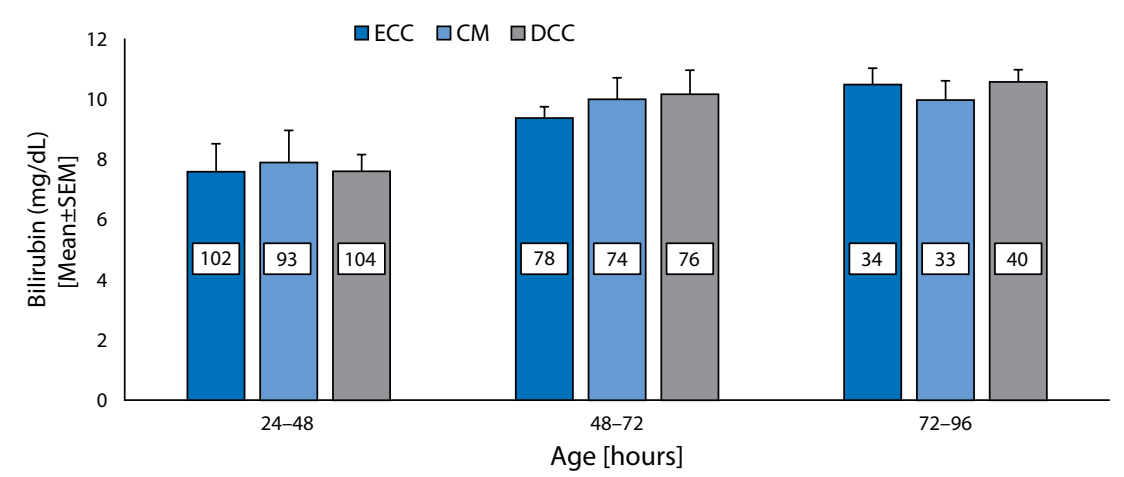

ECC-early cord clamping, CM-cord milking, DCC-delayed cord clamping

Figure 2. Transcutaneous bilirubin levels during the first 24-96 hours of life

deficiencies at ages 2 to 3 months in the DCC group [18-20]. Nonetheless, it is also important to understand, whether placental transfusion may also contribute for less favourable neonatal outcomes such as hyperbilirubinemia, (requiring phototherapy or exchange transfusion) and polycythaemia.

To our best knowledge, this is the first study, which was designed, to demonstrate that applying DCC or CM to term infants does not increase the risk of hyperbilirubinemia requiring phototherapy or exchange transfusion without harmful effects in comparison to ECC. The frequency of infants requiring phototherapy in CM or DCC groups was higher than in ECC group. However, due to small sample size, the non-inferiority analysis of the primary outcome was inconclusive. Perhaps, early cord clamping at only $30 \mathrm{sec}-$ onds (similarly to previous authors), would have provided a larger difference in outcome data [21].

Postnatal hyperbilirubinemia is universal and manifests as neonate jaundice in over $60-80 \%$ of all neonates [13]. If left untreated, hyperbilirubinemia may progress to excessive levels that may be associated with evident bilirubin 


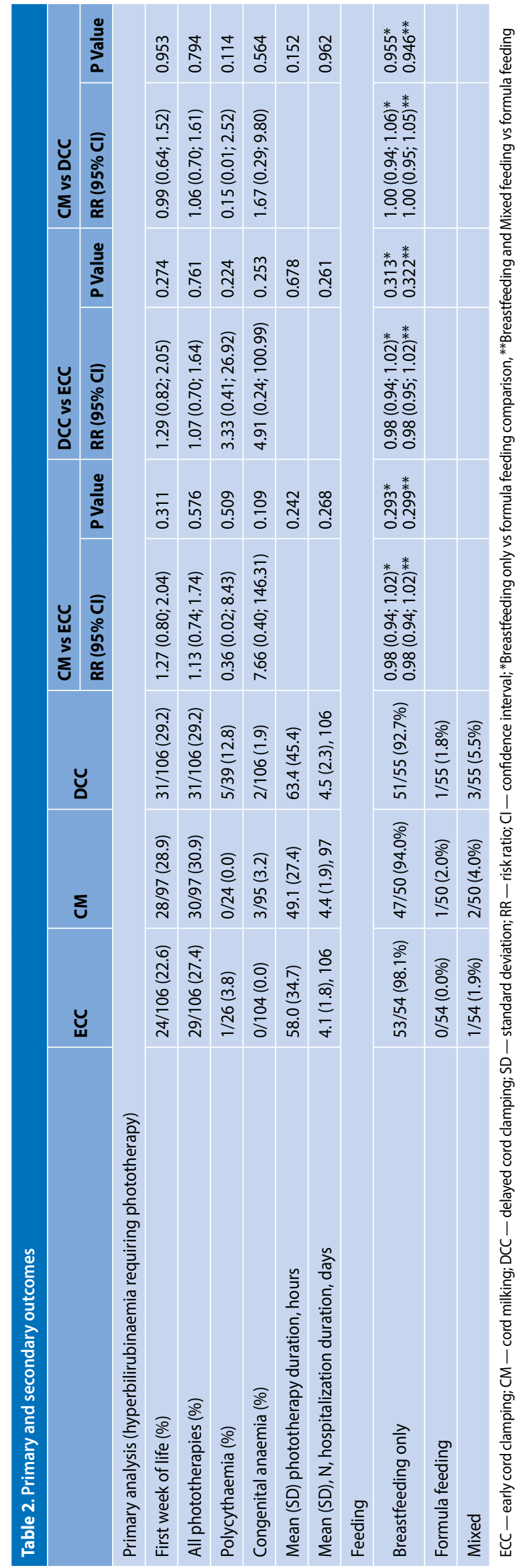

neurotoxicity. Available results from other studies regarding "jaundice" and "jaundice needing phototherapy" (associated with DCC) can be misleading. First, no information is offered on how "clinical jaundice" was assessed on examination, and estimation of the degree of hyperbilirubinemia based solely on clinical examination can lead to errors [22-24]. A review on DCC revealed only 4 studies which assessed polycythaemia and hyperbilirubinemia during the first week of life as a second objective [25]. No information was provided on which hour of life the bilirubin levels were measured exactly. In our study, to avoid loss or delay in diagnosis we screened all participants for jaundice using a bilirubinometer. Furthermore, guidelines to treat jaundice have changed over time and none of the studies mentioned what threshold was used for administering phototherapy. No information is given whether staff responsible for phototherapy administration was blinded to the type of cord clamping intervention.

In view of the decrease sample size, our results may underestimate the true prevalence of hyperbilirubinemia requiring phototherapy. Additional subgroup analyses to identify the risk of readmission secondary for jaundice, anaemia and iron storages at 3 months of age will be a subject of a separate publication. Possible confounders such as maternal pre-gestational diabetes, iso-immune haemolytic disease, were not included in the analysis, which may alter the results.

\section{CONCLUSIONS}

With early detection of hyperbilirubinemia and prompt initiation of treatment following accepted guidelines, a possible elevated risk of bilirubin encephalopathy can be minimized, while preserving all potential benefits of the placental transfusion in term infants.

\section{REFERENCES}

1. Wyllie J, Perlman JM, Kattwinkel J, et al. Neonatal Resuscitation Chapter Collaborators, Neonatal Resuscitation Chapter Collaborators, Neonatal Resuscitation Chapter Collaborators. Part 7: Neonatal Resuscitation: 2015 International Consensus on Cardiopulmonary Resuscitation and Emergency Cardiovascular Care Science With Treatment Recommendations (Reprint). Pediatrics. 2015; 136 Suppl 2(16 Suppl 1): S120-S166, doi: 10.1542/peds.2015-3373D, indexed in Pubmed: 26471381.

2. Prendiville WJ, Harding JE, Elbourne DR, et al. The Bristol third stage trial: active versus physiological management of third stage of labour. BMJ. 1988; 297(6659): 1295-1300, doi: 10.1136/bmj.297.6659.1295, indexed in Pubmed: 3144366.

3. McDonald SJ, Middleton P, Dowswell T, et al. Effect of timing of umbilical cord clamping of term infants on maternal and neonatal outcomes. Cochrane Database Syst Rev. 2008; 9(2): CD004074-397, doi: 10.1002/14651858.CD004074.pub2, indexed in Pubmed: 18425897.

4. Saigal S, O'Neill A, Surainder Y, et al. Placental transfusion and hyperbilirubinemia in the premature. Pediatrics. 1972; 49(3): 406-419, indexed in Pubmed: 5062269.

5. Hutton EK, Hassan ES. Late vs early clamping of the umbilical cord in full-term neonates: systematic review and meta-analysis of controlled trials. JAMA. 2007; 297(11): 1241-1252, doi: 10.1001/jama.297.11.1241, indexed in Pubmed: 17374818.

6. Tarnow-Mordi W, Morris J, Kirby A, et al. Australian Placental Transfusion Study Collaborative Group. Delayed versus Immediate Cord Clamp- 
ing in Preterm Infants. N Engl J Med. 2017; 377(25): 2445-2455, doi: 10.1056/NEJMoa1711281, indexed in Pubmed: 29081267.

7. Fogarty $M$, Osborn DA, Askie $L$, et al. Delayed vs early umbilical cord clamping for preterm infants: a systematic review and meta-analysis. Am J Obstet Gynecol. 2018; 218(1): 1-18, doi: 10.1016/j.ajog.2017.10.231, indexed in Pubmed: 29097178.

8. Yang S, Duffy JY, Johnston R, et al. Association of a Delayed Cord-Clamping Protocol With Hyperbilirubinemia in Term Neonates. Obstet Gynecol. 2019; 133(4): 754-761, doi: 10.1097/AOG.0000000000003172, indexed in Pubmed: 30870273.

9. Linderkamp $O$, Nelle $M$, Kraus $M$, et al. The effect of early and late cord-clamping on blood viscosity and other hemorheological parameters in full-term neonates. Acta Paediatr. 1992; 81(10): 745-750, doi: 10.1111/j.1651-2227.1992.tb12095.x, indexed in Pubmed: 1421876.

10. Yao $A C L$, Vuorenkoski J. Expiratory grunting in the late clamped normal neonate. Pediatrics. 1971; 48(6): 865-870.

11. Rabe H, Jewison A, Alvarez RF, et al. Brighton Perinatal Study Group. Milking compared with delayed cord clamping to increase placental transfusion in preterm neonates: a randomized controlled trial. Obstet Gynecol. 2011; 117(2 Pt 1):205-211, doi: 10.1097/AOG.0b013e3181fe46ff, indexed in Pubmed: 21252731.

12. Brune T, Garritsen H, Witteler R, et al. Autologous placental blood transfusion for the therapy of anaemic neonates. Biol Neonate. 2002; 81(4): 236-243, doi: 10.1159/000056754, indexed in Pubmed: 12011567.

13. Bhutani VK, Committee onF. Neonate, American Academy of P. Phototherapy to prevent severe neonatal hyperbilirubinemia in the neonate infant 35 or more weeks of gestation. Pediatrics. 2011; 128(4): e1046-52.

14. Emhamed MO, van Rheenen $P$, Brabin BJ. The early effects of delayed cord clamping in term infants born to Libyan mothers. Trop Doct. 2004; 34(4): 218-222, doi: 10.1177/004947550403400410, indexed in Pubmed: 15510946.

15. American Academy of Pediatrics Subcommittee on Hyperbilirubinemia. Management of hyperbilirubinemia in the newborn infant 35 or more weeks of gestation. Pediatrics. 2004; 114(1): 297-316, doi: 10.1542/peds.114.1.297, indexed in Pubmed: 15231951.

16. Qian $Y$, Ying $X$, Wang $P$, et al. Effect of timing of umbilical cord clamping of term infants on maternal and neonatal outcomes. Cochrane Data- base Syst Rev. 2008; 112(2): CD004074-178, doi: 10.1002/14651858. CD004074.pub2, indexed in Pubmed: 18425897.

17. Maisels MJ, Kring E. Transcutaneous bilirubin levels in the first 96 hours in a normal newborn population of $>$ or $=35$ weeks' gestation. Pediatrics. 2006; 117(4): 1169-1173, doi: 10.1542/peds.2005-0744, indexed in Pubmed: 16585312

18. Gupta R, Ramji S. Effect of delayed cord clamping on iron stores in infants born to anemic mothers: a randomized controlled trial. Indian Pediatr. 2002; 39(2): 130-135, indexed in Pubmed: 11867842.

19. Grajeda R, Pérez-Escamilla R, Dewey KG. Delayed clamping of the umbilical cord improves hematologic status of Guatemalan infants at 2 mo of age. Am J Clin Nutr. 1997; 65(2): 425-431, doi: 10.1093/ajcn/65.2.425, indexed in Pubmed: 9022526.

20. Hutton EK, Hassan ES. Late vs early clamping of the umbilical cord in full-term neonates: systematic review and meta-analysis of controlled trials. JAMA. 2007; 297(11): 1241-1252, doi: 10.1001/jama.297.11.1241, indexed in Pubmed: 17374818.

21. Rabe H, Jewison A, Alvarez RF, et al. Brighton Perinatal Study Group. Milking compared with delayed cord clamping to increase placental transfusion in preterm neonates: a randomized controlled trial. Obstet Gynecol. 2011;117(2 Pt 1): 205-211, doi: 10.1097/AOG.0b013e3181fe46ff, indexed in Pubmed: 21252731.

22. Keren $\mathrm{R}$, Luan $\mathrm{X}$, Friedman $\mathrm{S}$, et al. A comparison of alternative risk-assessment strategies for predicting significant neonatal hyperbilirubinemia in term and near-term infants. Pediatrics. 2008; 121(1): e170-e179, doi: 10.1542/peds.2006-3499, indexed in Pubmed: 18166536.

23. Johnson L, Bhutani V. Guidelines for Management of the Jaundiced Term and Near-Term Infant. Clinics in Perinatology. 1998; 25(3): 555-574, doi: 10.1016/s0095-5108(18)30097-6.

24. Bhutani VK, Johnson LH, Keren R. Diagnosis and management of hyperbilirubinemia in the term neonate: for a safer first week. Pediatr Clin North Am. 2004; 51(4): 843-61, vii, doi: 10.1016/j.pcl.2004.03.011, indexed in Pubmed: 15275978.

25. van Rheenen $P$, Brabin BJ. Late umbilical cord-clamping as an intervention for reducing iron deficiency anaemia in term infants in developing and industrialised countries: a systematic review. Ann Trop Paediatr. 2004; 24(1): 3-16, doi: 10.1179/027249304225013286, indexed in Pubmed: 15005961. 